\title{
Serum Osteopontin as a Novel Biomarker for Muscle Regeneration in Duchenne Muscular Dystrophy
}

\author{
Mutsuki Kuraoka, ${ }^{*}$ En Kimura, ${ }^{* \dagger}$ Tetsuya Nagata, ${ }^{\star \ddagger}$ Takashi Okada, ${ }^{\S}$ Yoshitsugu Aoki, ${ }^{*}$ Hisateru Tachimori, ${ }^{\natural}$ \\ Naohiro Yonemoto," Michihiro Imamura, ${ }^{*}$ and Shin'ichi Takeda*
}

\begin{abstract}
From the Department of Molecular Therapy, * National Institute of Neuroscience, the Translational Medical Center, ${ }^{\dagger}$ and the Departments of Mental Health Policy and Evaluation" and Psychopharmacology," National Institute of Mental Health, National Center of Neurology and Psychiatry, Tokyo; the Department of Neurology and Neurological Science, ${ }^{\ddagger}$ Graduate School of Medicine, Tokyo Medical and Dental University, Tokyo; and the Department of Biochemistry and Molecular Biology, ${ }^{\S}$ Nippon Medical School, Tokyo, Japan
\end{abstract}

Accepted for publication January 5, 2016.

Address correspondence to Shin'ichi Takeda, M.D., Ph.D., Department of Molecular Therapy, National Institute of Neuroscience, National Center of Neurology and Psychiatry, 4-1-1 Ogawa-Higashi, Kodaira, Tokyo 187-8502, Japan. E-mail: takeda@ncnp.go.jp.

\begin{abstract}
Duchenne muscular dystrophy is a lethal X-linked muscle disorder. We have already reported that osteopontin (OPN), an inflammatory cytokine and myogenic factor, is expressed in the early dystrophic phase in canine X-linked muscular dystrophy in Japan, a dystrophic dog model. To further explore the possibility of OPN as a new biomarker for disease activity in Duchenne muscular dystrophy, we monitored serum OPN levels in dystrophic and wild-type dogs at different ages and compared the levels to other serum markers, such as serum creatine kinase, matrix metalloproteinase-9, and tissue inhibitor of metalloproteinase-1. Serum OPN levels in the dystrophic dogs were significantly elevated compared with those in wild-type dogs before and 1 hour after a cesarean section birth and at the age of 3 months. The serum OPN level was significantly correlated with the phenotypic severity of dystrophic dogs at the period corresponding to the onset of muscle weakness, whereas other serum markers including creatine kinase were not. Immunohistologically, OPN was up-regulated in infiltrating macrophages and developmental myosin heavy chain-positive regenerating muscle fibers in the dystrophic dogs, whereas serum OPN was highly elevated. OPN expression was also observed during the synergic muscle regeneration process induced by cardiotoxin injection. In conclusion, OPN is a promising biomarker for muscle regeneration in dystrophic dogs and can be applicable to boys with Duchenne muscular dystrophy. (Am J Pathol 2016, 186: 1302-1312; http://dx.doi.org/10.1016/j.ajpath.2016.01.002)
\end{abstract}

Duchenne muscular dystrophy (DMD) is an X-linked disorder of muscle characterized by primary muscle atrophy. ${ }^{1}$ Its prevalence in the population is estimated to be 1 in 3500 male newborns. ${ }^{2}$ A $D M D$ gene mutation results in the absence of dystrophin, a structural protein in muscle fibers, leading to fragility of muscle fibers to contractive force. ${ }^{3}$ Histologic features of DMD are muscle fiber degeneration with secondary cellular inflammation, ineffective muscle fiber regeneration, and eventually fibrosis and adiposis. Hence, patients with DMD have muscle weakness, leading to loss of ambulation and early death from respiratory or cardiac failure.

Clinical biomarkers are necessary for the diagnosis of the disease, classification of the severity, and evaluation of therapeutic effects. Serum creatine kinase (CK) is a primary biomarker for the sensitive diagnosis of DMD, reflecting muscle damage. ${ }^{4}$ However, serum CK is not sufficient for the evaluation of therapeutic effects because its levels decrease with the progression of dystrophic disease corresponding to skeletal muscle waste. Osteopontin $(\mathrm{OPN})$ is a potential new candidate for development of a method for evaluation of dystrophic conditions. OPN is a

Supported by Neurological and Psychiatric Disorders of the National Center of Neurology and Psychiatry intramural research grants 22-5 and 255 (S.T.) and Japan Society for the Promotion of Science Grants-in-Aid for Scientific Research grant 24591284 (E.K.).

Disclosures: None declared. 
phosphorylated glycoprotein that is synthesized in a variety of tissues and cells, and participates in inflammation, tissue remodeling, and tumorigenesis. ${ }^{5,6}$ The secreted form of OPN, both as a soluble molecule with cytokine functions and as an immobilized matricellular protein, can interact with CD44 and a variety of integrins to activate intracellular signaling pathways and mediate cell-cell and cellmatrix interactions. ${ }^{5,7}$ The effects of binding of OPN to target cells, including promotion of attachment, proliferation, migration, and chemotaxis of cells, are modulated through cleavage by thrombin and matrix metalloproteinase (MMP)-3, -7, and $-12 .{ }^{8,9}$ In injured or dystrophic muscles, OPN is reportedly expressed in immune cells, myoblasts, and damaged or regenerating muscle fibers, indicating its functional involvement in cellular inflammation, muscle regeneration, and fibrosis. ${ }^{10-16}$ Serum OPN level elevation is also observed in X-linked muscular dystrophy ( $\mathrm{mdx}$ ) mice. ${ }^{13}$ OPN may influence the severity of disease in patients with DMD because single-nucleotide polymorphisms in human SPP1 $(O P N)$ correlate with the results of clinical trials. ${ }^{17-19}$ In our previous study using a dystrophin-deficient dog model, canine X-linked muscular dystrophy in Japan $\left(\mathrm{CXMD}_{\mathrm{J}}\right)$, we found that OPN is implicated in dystrophic diaphragms at birth before initial respiration, suggesting the participation of OPN in a dystrophic muscle environment even at an early phase. ${ }^{20}$

In a recent study of other serum biomarkers for which the biological function may be related to that of OPN, MMP-9 and its endogenous inhibitor, tissue inhibitor of metalloproteinase (TIMP)-1, are strongly suggested to be DMD biomarkers. ${ }^{21}$ MMP-9 is a proteolytic enzyme that degrades extracellular matrix components and is prominently involved in the inflammatory process during muscle degeneration. ${ }^{22-24}$ TIMP-1 possesses biological properties independent of MMP inhibitory activity, including stimulation of cell proliferation and antiapoptosis. ${ }^{25,26}$ Elevation of serum MMP-9 and TIMP-1 levels is associated with dystrophic disease, and MMP-9 levels correlate with clinical nonambulation of patients with DMD. ${ }^{21}$

We hypothesized that OPN is uniquely expressed at the early dystrophic phase, and could therefore be a new biomarker of DMD. However, what the serum OPN level indicates in dystrophic disease is still unclear because its elevation is not constantly observed during disease progression. ${ }^{13,21}$ In the present study, we analyzed serum OPN levels in $\mathrm{CXMD}_{\mathrm{J}}$ dystrophic dogs and wild-type (WT) controls at birth and during growth periods and compared them with levels of serum CK, MMP-9, and TIMP-1. We report here for the first time that the serum OPN level in dystrophic dogs was significantly higher than in WT at birth and in the active muscle regeneration phase. OPN was expressed in infiltrating immune cells and regenerating muscle fibers in dystrophic muscles, suggesting an association with elevated serum OPN levels.

\section{Materials and Methods}

\section{Animals}

A $\mathrm{CXMD}_{\mathrm{J}}$ dog colony was established by insemination of beagles with the sperm of golden retriever muscular dystrophy (GRMD) dogs. ${ }^{27} \mathrm{CXMD}_{\mathrm{J}}$ dystrophic dogs lack dystrophin in the muscle tissue and have dystrophic phenotypes, as observed in GRMD and in human DMD. ${ }^{28}$ All the animals were cared for and treated in accordance with the guidelines provided by the Ethics Committee for the Treatment of Laboratory Middlesized Animals of the National Institute of Neuroscience. Forty-nine $\mathrm{CXMD}_{\mathrm{J}}$ dystrophic and $40 \mathrm{WT}$ littermates in the second to seventh generations from the first artificial insemination were used in this study. All the serum and muscle samples in this study were routinely taken from the dystrophic and WT dogs raised between September 2002 and September 2014 except the ones used for other interventional studies. Anesthesia in the dystrophic and WT dogs was induced by intravenous injection of $20 \mathrm{mg} / \mathrm{kg}$ of thiopental sodium (Ravonal, Mitsubishi Tanabe Pharma, Osaka, Japan) and maintained by inhalation of isoflurane (Isoflu, DS Pharma Animal Health Co., Osaka, Japan). Euthanization was conducted by exsanguination under the anesthetic condition.

\section{Serum Collection and Serum Chemistry}

Blood samples were collected from each dog at birth, at the age of 3 and 6 weeks, at 2, 3, 6, and 9 months, and at 1 and 2 years. Umbilical cord blood was obtained after fetuses were expelled with a cesarean section, and blood was withdrawn from all the neonates 1 hour after the initial respiration. ${ }^{20}$ Each blood sample was treated and assayed for serum CK activity using an automated colorimetric analyzer (FDC3500, Fuji Film Medical Co. Ltd., Tokyo, Japan), as previously reported. ${ }^{29}$

\section{Enzyme-Linked Immunosorbent Assay Analysis}

Serum OPN, MMP-9, and TIMP-1 levels were determined using an enzyme-linked immunosorbent assay (ELISA) kit (USCN Life Science Inc., Wuhan, China) for Canis familiaris OPN (E90899Ca), MMP-9 (E90553Ca), and TIMP-1 (E90552Ca). Sample application was performed in duplicate according to the manufacturer's instructions. Optical density values of standards and samples were spectrophotometrically measured at a wavelength of $450 \mathrm{~nm}$ using a microplate reader (Appliskan, Thermo Fisher Scientific, Waltham, MA). When the values were below the detection limit, they were assigned a value of zero.

\section{Clinical Manifestations}

Clinical evaluation of dystrophic dogs as described in our previous reports ${ }^{28-30}$ was performed with revision of some of the items. Briefly, we evaluated gait and mobility disturbances, limb and temporal muscle atrophy, drooling, macroglossia, 

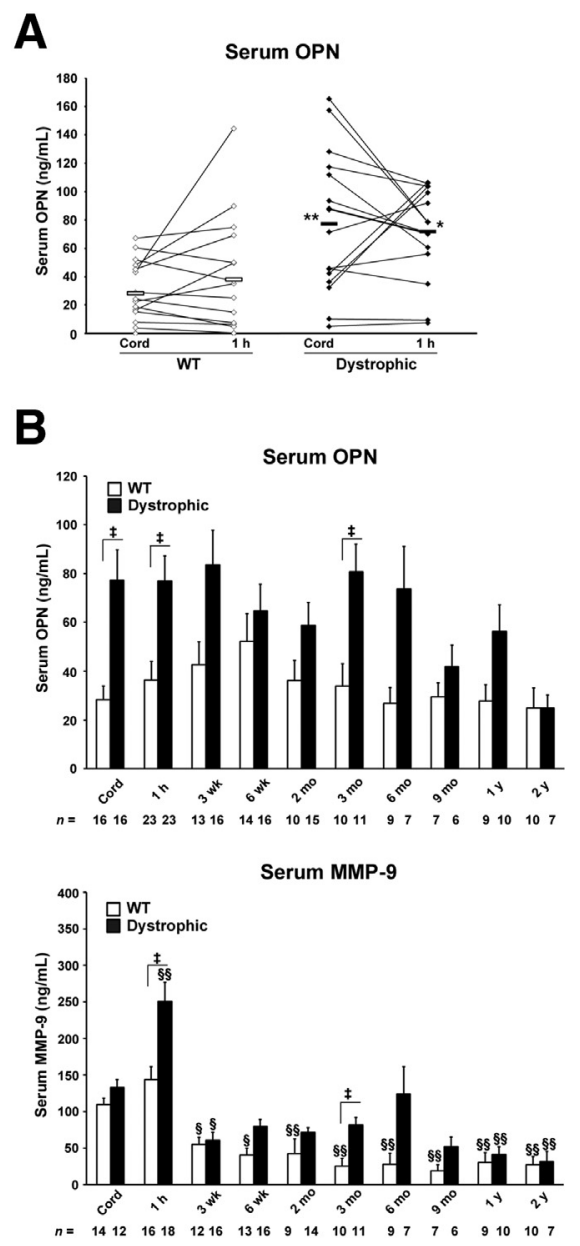
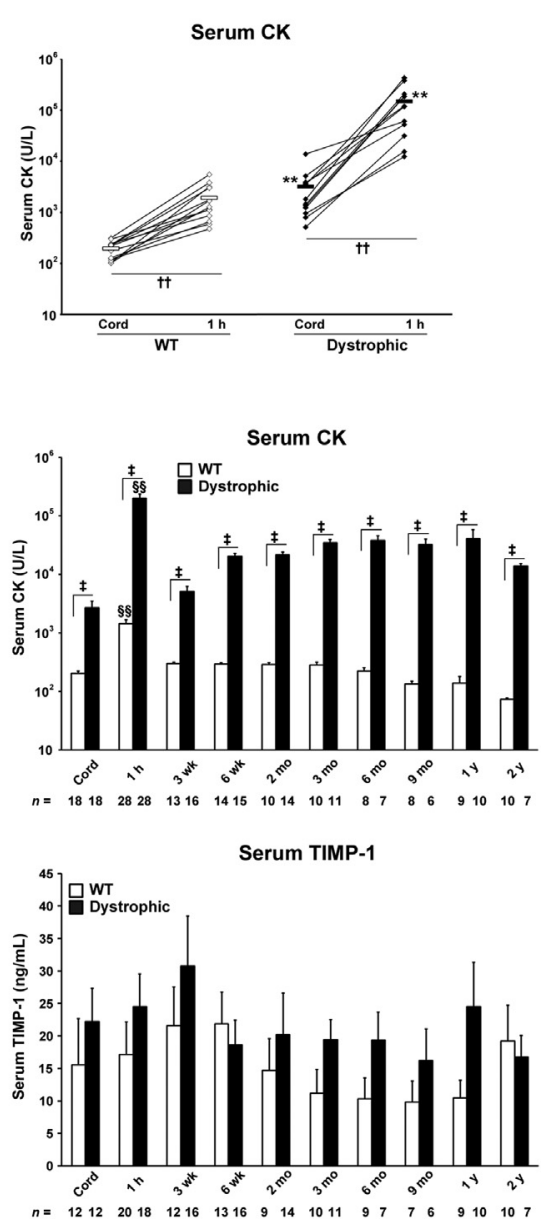

Figure 1 Patterns of elevation of serum osteopontin (OPN), creatine kinase (CK), matrix metalloproteinase (MMP)-9, and tissue inhibitor of metalloproteinase (TIMP)-1 in wild-type (WT) and dystrophic dogs. A: Paired serum OPN and CK levels in WT and dystrophic dogs before and after birth. Cord and 1 hour indicate serum obtained before and after birth, respectively. Bars indicate the mean value. B: Serum OPN, CK, MMP-9, and TIMP-1 levels at the different ages. The data are the means \pm SEM. $n=16$ for WT and dystrophic dogs in serum OPN $(\mathbf{A}) ; \mathrm{n}=13$ for WT dogs in serum CK $(\mathbf{A}) ; n=12$ for dystrophic dogs in serum CK (A). ${ }^{*} P<0.05$ and ${ }^{* *} P<0.01$ versus WT using $t$-test; ${ }^{\dagger \dagger} P<0.01$ versus cord values in WT using paired $t$-test in $\mathbf{A} .{ }^{\ddagger} P<0.05$ versus WT using $t$-test with the Holm multiple test; ${ }^{\S} P<0.05$ and ${ }^{\S \S} P<$ 0.01 compared between values of the cord and each age point in WT or dystrophic dogs using Dunnett's test in B. dysphagia, and abnormal sitting posture as clinical signs. The severity of each sign was classified into scores of 1 to 5 (grade 1, none; grade 5, severe) according to a grading scale for $\mathrm{CXMD}_{\mathrm{J}}$ (Supplemental Table S1). We used the grading records between October 2007 and May 2013.

\section{Histopathology and Immunohistochemistry}

The diaphragm and tibialis cranialis (TC) muscle tissues were sampled by autopsy, as previously described. ${ }^{20,29,31}$ Briefly, muscle tissues were snap-frozen in cooled isopentane and stored at $-80^{\circ} \mathrm{C}$ before use. Cryostat sections $(8 \mu \mathrm{m})$ were serially prepared and routinely stained with hematoxylin and eosin.

Immunostaining was performed on serial sections, as previously described. ${ }^{20}$ Briefly, all sections were fixed in cooled acetone and blocked with Block Ace (Dainippon Sumitomo
Pharma Co., Ltd., Osaka, Japan). Subsequently, the specimens were labeled with primary rabbit polyclonal antibodies to OPN (dilution, 1:100) (RB-9097, Thermo Fisher Scientific), MMP-9 (dilution, 1:1000) (ab38898, Abcam, Cambridge, UK), or TIMP-1 (dilution, 1:200) (AB770, EMD Millipore Corp., Temecula, CA) and with mouse monoclonal antibodies to developmental myosin heavy chain (dMyHC) (dilution, 1:40) (NCL-MHCd, Leica Biosystems, Newcastle, UK), CD11b (dilution, 1:50) (MCA1777S, AbD Serotec, Kidlington, Oxford, UK), CD3 (dilution, 1:50) (MCA1774GA, AbD Serotec), CD18 (dilution, 1:10) (MCA1780, AbD Serotec), CD68 (dilution, 1:100) (MCA1815T, AbD Serotec), or CD206 (dilution, 1:50) (MCA2519GA, AbD Serotec). Secondary antibodies used were fluorescein-conjugated goat anti-rabbit or anti-mouse IgG (Alexa Fluor 488 or 568, respectively; dilution, 1:1000; Life Technologies, Carlsbad, CA). All sections were

Table 1 Correlation between Serum Biomarker Levels and Total Clinical Scores in Dystrophic Dogs at the Age of 2 Months

\begin{tabular}{lllll}
\hline Variable & Osteopontin & CK & MMP-9 & TIMP-1 \\
\hline$n$ & 11 & 11 & 10 & 9 \\
Pearson correlation coefficient & $0.672^{*}$ & 0.221 & -0.132 & 0.485 \\
\hline
\end{tabular}

Data with missing values were removed from the analysis.

${ }^{*} P<0.05$.

CK, creatine kinase; MMP-9, matrix metalloproteinase-9; TIMP-1, tissue inhibitor of metalloproteinase-1. 


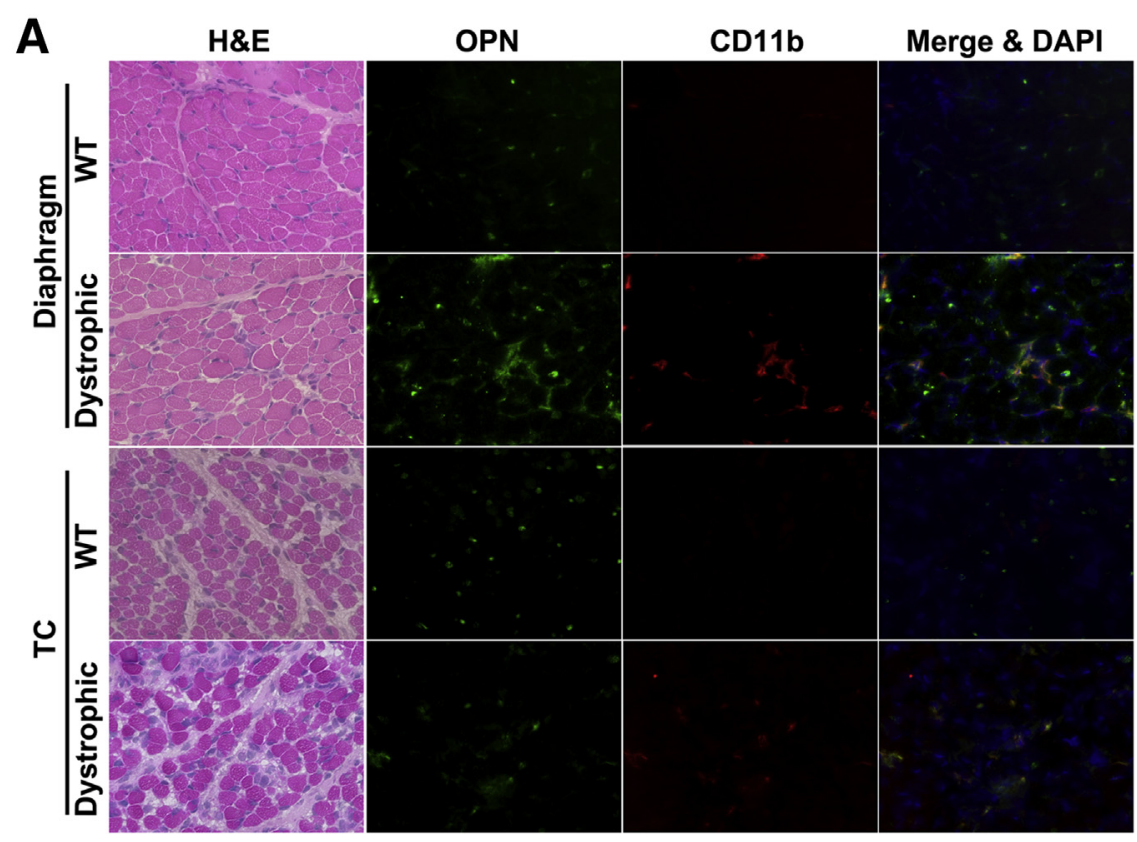

B

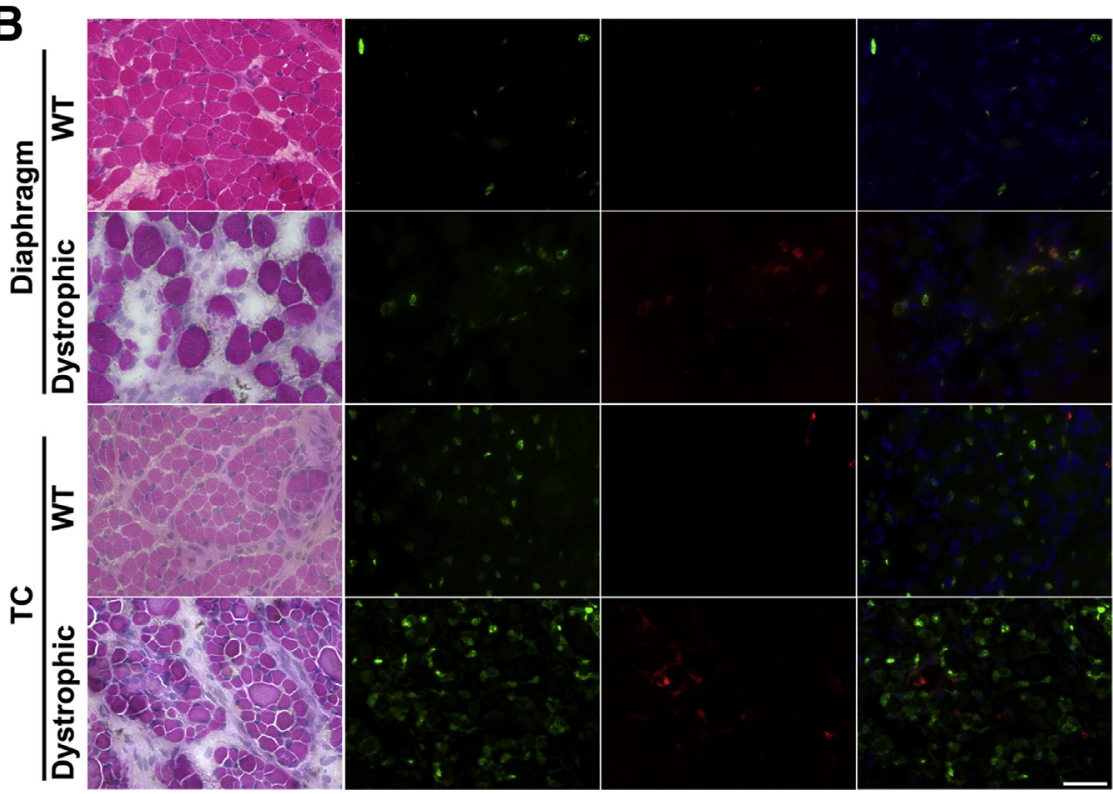

Figure 2 0steopontin (OPN) expression in infiltrating macrophages of dystrophic muscles at birth. Serial sections of wild-type (WT) and dystrophic diaphragm and tibialis cranialis (TC) muscles before (A) and after (B) initial respiration are stained with hematoxylin and eosin (H\&E) or immunostained for OPN (green) and CD11b (red). Merged immunostained images co-stained with DAPI (blue; nucleus) are also shown. Scale bar $=$ $40 \mu \mathrm{m}$. mounted with fluorescent mounting medium containing DAPI (Vectashield, Vector Laboratories Inc., Burlingame, CA). Images were captured with a fluorescence microscope BZ9000 (Keyence, Osaka, Japan) under identical conditions.

\section{Muscle Fiber Counting}

All fluorescent images were analyzed with a computer-aided image analysis system (BZ-II Analyzer 1.41, Keyence). Muscle fiber counting was performed to compare the population of OPN- and/or dMyHC-positive muscle fibers. In the dystrophic diaphragm and TC muscle tissues, OPN- and/or dMyHCpositive muscle fibers were counted after removal of weakly positive fibers and were compared at the age of 2 to 4 weeks, 3 to 5 months, and 1 and 2 to 4 years. In each section, four random fields (in original magnification $\times 20$ ) were selected per specimen. OPN- and dMyHC-positive or -negative fibers in these fields were manually captured, and the number was measured. The total number of muscle fibers was measured with bright images in which we were able to capture fiber outlines. The percentage of positive muscle fibers was calculated as follows:

Total Number of Positive Muscle Fibers/Total Number of Muscle Fibers $\times 100$ (Total Numbers Were Summed From Four Fields).

\section{Cardiotoxin Injection}

Nine WT dogs at the age of 3 to 4.5 months were anesthetized and injected with cardiotoxin (a venom from Naja mossambica mossambica, $50 \mu \mathrm{mol} / \mathrm{L}, 0.21 \mathrm{~mL} / \mathrm{kg}$ ) (C9759, Sigma-Aldrich, 


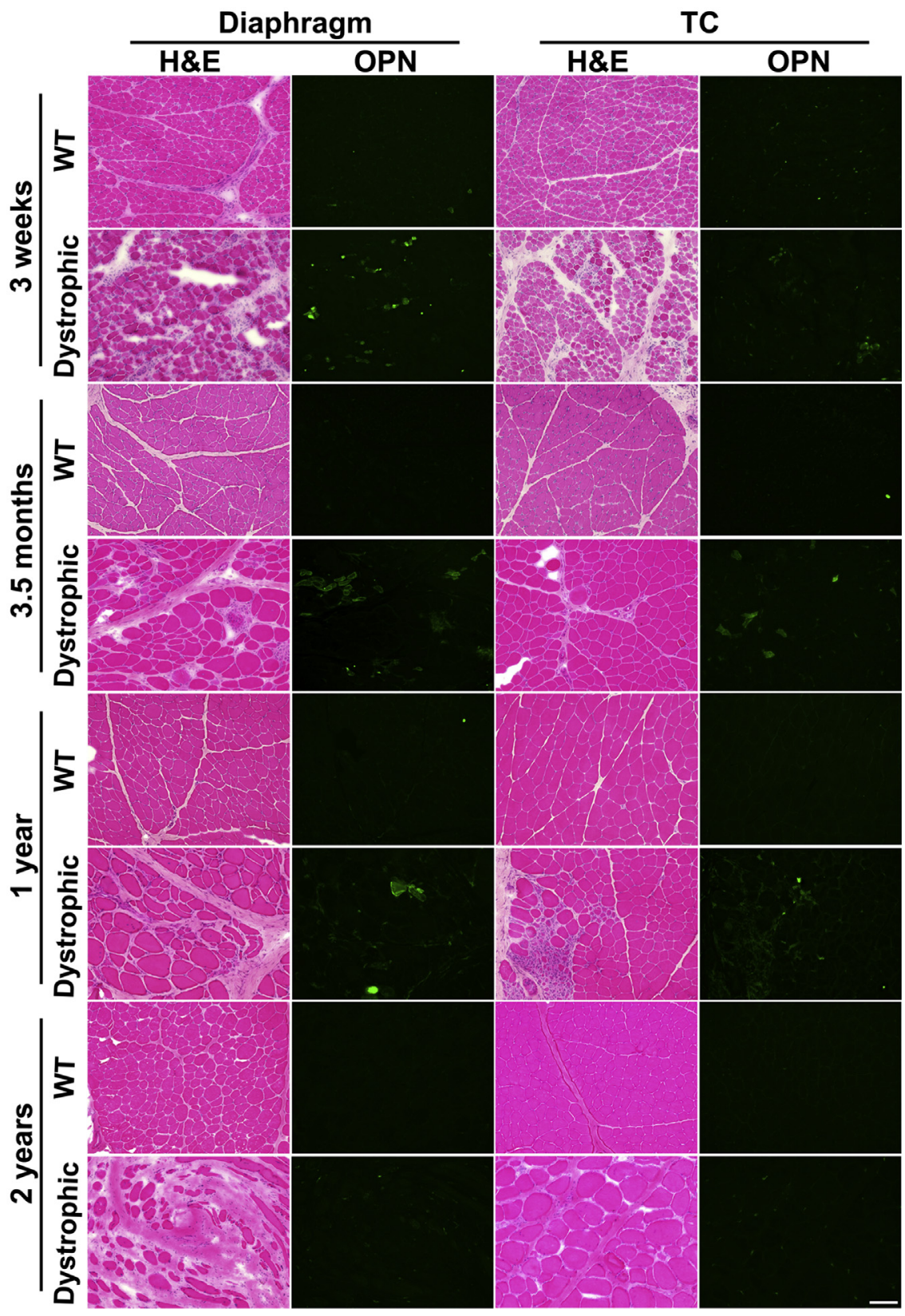

Figure 3 0steopontin (OPN) expression in dystrophic muscles at the age of 3 weeks, 3.5 months, and 1 and 2 years. Serial sections of diaphragm and tibialis cranialis (TC) muscles of wild-type (WT) and dystrophic dogs are stained with hematoxylin and eosin (H\&E) and immunostained for OPN (green). Muscles from dogs at the age of 3 weeks, 3.5 months, and 1 and 2 years were stained. Scale bar $=100 \mu \mathrm{m}$.

St. Louis, MO) into the bilateral TC muscles to induce muscle injury and subsequent regeneration. ${ }^{10}$ For analgesia treatment, $0.02 \mathrm{mg} / \mathrm{kg}$ of buprenorphine hydrochloride (Lepetan, Otsuka Pharmaceutical, Tokyo, Japan) was injected i.m. before the dogs awoke from general anesthesia. Blood samples were collected before injection and 1, 3, 5, and 7 days after injection. Three dogs were euthanized and the bilateral TC muscles removed at 3, 5, and 7 days after injection, respectively. Serum $\mathrm{CK}$ and OPN measurement and immunohistological analysis were performed as described above.

\section{Statistical Analysis}

For analysis of serum before and after birth, biomarker levels were compared with a paired $t$-test, and those between WT and dystrophic dogs were compared with an unpaired $t$-test. For serum level analyses at different ages, the Holm multiple test ${ }^{32,33}$ was applied in multiple comparisons between WT and dystrophic levels at all age points. In addition, serum biomarker levels at each age point were compared with the prebirth level with Dunnett's test. For analysis of cardiotoxin injection, serum biomarker levels on each postinjection day were compared with the preinjection level in the same animals using a paired $t$-test, and the Holm multiple test was used at all postinjection days. For analysis of the percentage of immunopositive muscle fibers, the percentages of OPN- and/or dMyHCpositive muscle fibers in each age range were calculated, and the median values were compared to those at the age of 2 to 4 years using the Mann-Whitney $U$ test with 
Bonferroni correction. Pearson's correlation was used for correlation studies. The statistical significance level was set at $5 \%$. In the Holm multiple test, ${ }^{32}$ the tests were ordered from those with the smallest $P$ value to the largest $P$ value. The test with the lowest probability was then tested first, followed by Bonferroni correction for all tests. The second test was tested with Bonferroni correction involving one less test and so on for the remaining tests. All data are given as the means \pm SEM, unless otherwise noted. All calculations were performed with StatView-J software version 5.0 (SAS Institute Inc., Cary, NC).

\section{Results}

\section{Analyses of Serum OPN and CK Levels in Dystrophic Dogs at Birth}

Serum OPN and CK levels in $\mathrm{CXMD}_{\mathrm{J}}$ dystrophic and WT littermates were analyzed using cord blood samples before and 1 hour after birth in a parametric manner (Figure 1A). Serum OPN levels in dystrophic dogs were significantly higher both before and after birth than those of WT dogs. Serum OPN levels were not significantly different between before and after birth in either WT or dystrophic dogs. On the other hand, serum CK levels in dystrophic dogs were significantly higher before and after birth than those of WT, and the postbirth level was drastically increased compared with before birth.

\section{Analyses of Serum Biomarker Levels in Dystrophic Dogs during Growth Periods}

Serum OPN, CK, MMP-9, and TIMP-1 levels were examined at different ages during growth periods in a nonparametric manner (Figure 1B). Serum OPN levels in dystrophic dogs were significantly higher than those in WT before and 1 hour after birth and at the age of 3 months. The serum OPN level at the age of 3 weeks remained higher but was not statistically significant compared with that of WT.

Serum CK levels in dystrophic dogs were significantly higher than those in WT at all age points (Figure 1B). The serum CK level in dystrophic dogs transiently decreased at the age of 3 weeks and then progressively increased again until the age of 3 months.

Serum MMP-9 levels in dystrophic dogs were significantly higher at 1 hour after birth and the age of 3 months than those in WT (Figure 1B). The serum MMP-9 level was significantly increased at 1 hour after birth but was then decreased at the age of 3 weeks compared with the prebirth value. Serum TIMP-1 levels in dystrophic dogs were not significantly different compared with those in WT dogs at any of the age points (Figure 1B). The TIMP1 levels were slightly increased before and 1 hour after birth and at the age of 3 weeks compared with those of WT dogs.
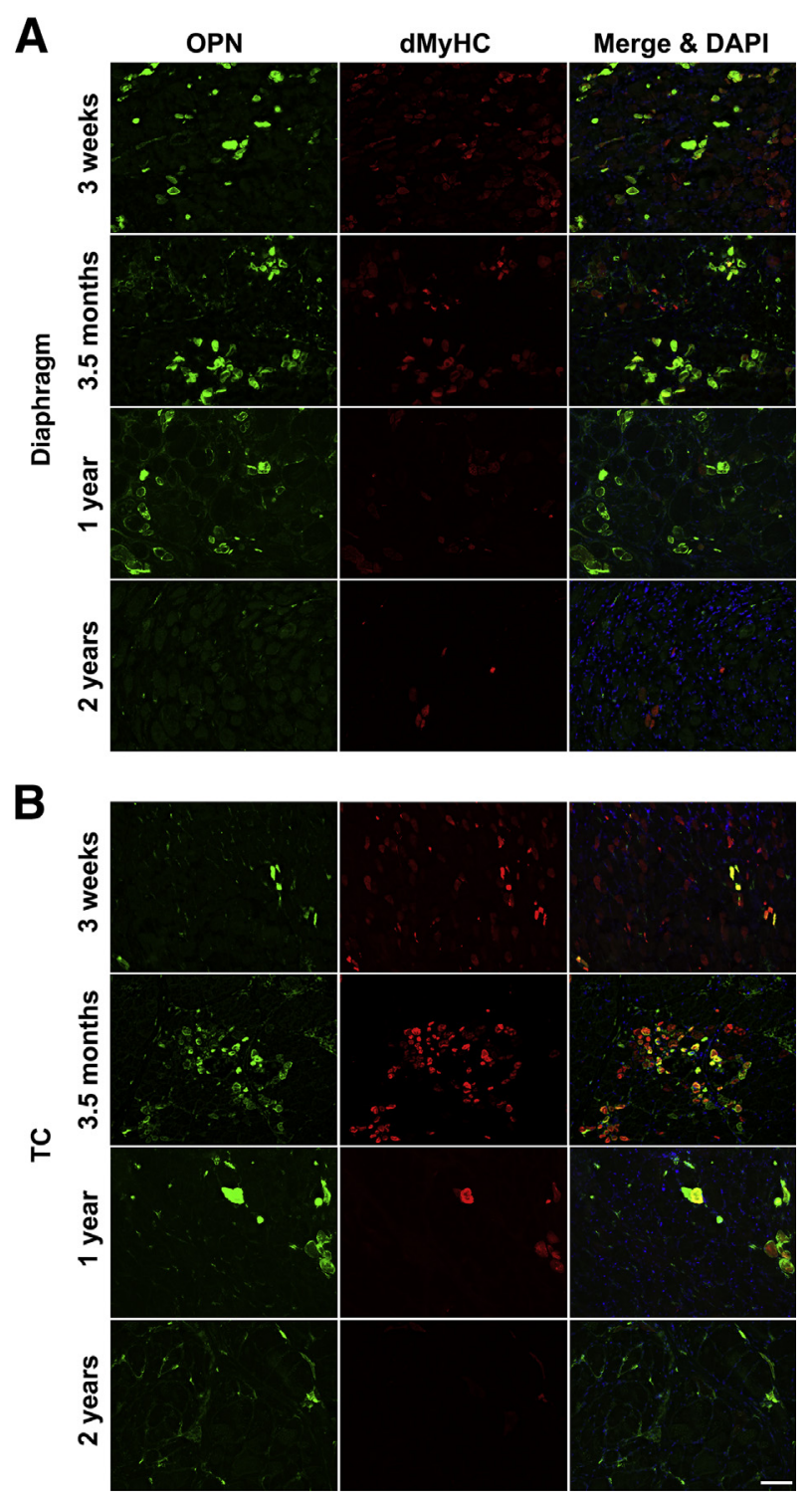

Figure 4 0steopontin (OPN) expression in regenerating muscle fibers of dystrophic muscles. Sections of dystrophic diaphragm (A) and tibialis cranialis (TC) (B) muscles are immunostained for OPN (green) and developmental myosin heavy chain ( $\mathrm{dMyHC}$, red) at the age of 3 weeks, 3.5 months, and 1 and 2 years. Merged images co-stained with DAPI (blue; nucleus) are shown on the right. Higher magnification images are shown in Supplemental Figure S2. Scale bar $=100 \mu \mathrm{m}$.

\section{Analyses of Serum Biomarker Levels and Phenotypic Severity in Dystrophic Dogs}

We analyzed whether serum biomarkers, including OPN, were correlated with phenotypic severity in dystrophic dogs. For classification of phenotypic variation, clinical manifestations were investigated in dystrophic dogs at the age of 2 months, when they had onset of clinical signs. ${ }^{28} \mathrm{We}$ found a significant correlation between the total grading score and the serum OPN level, whereas no correlation was found between the score and other markers, including serum CK (Table 1). 

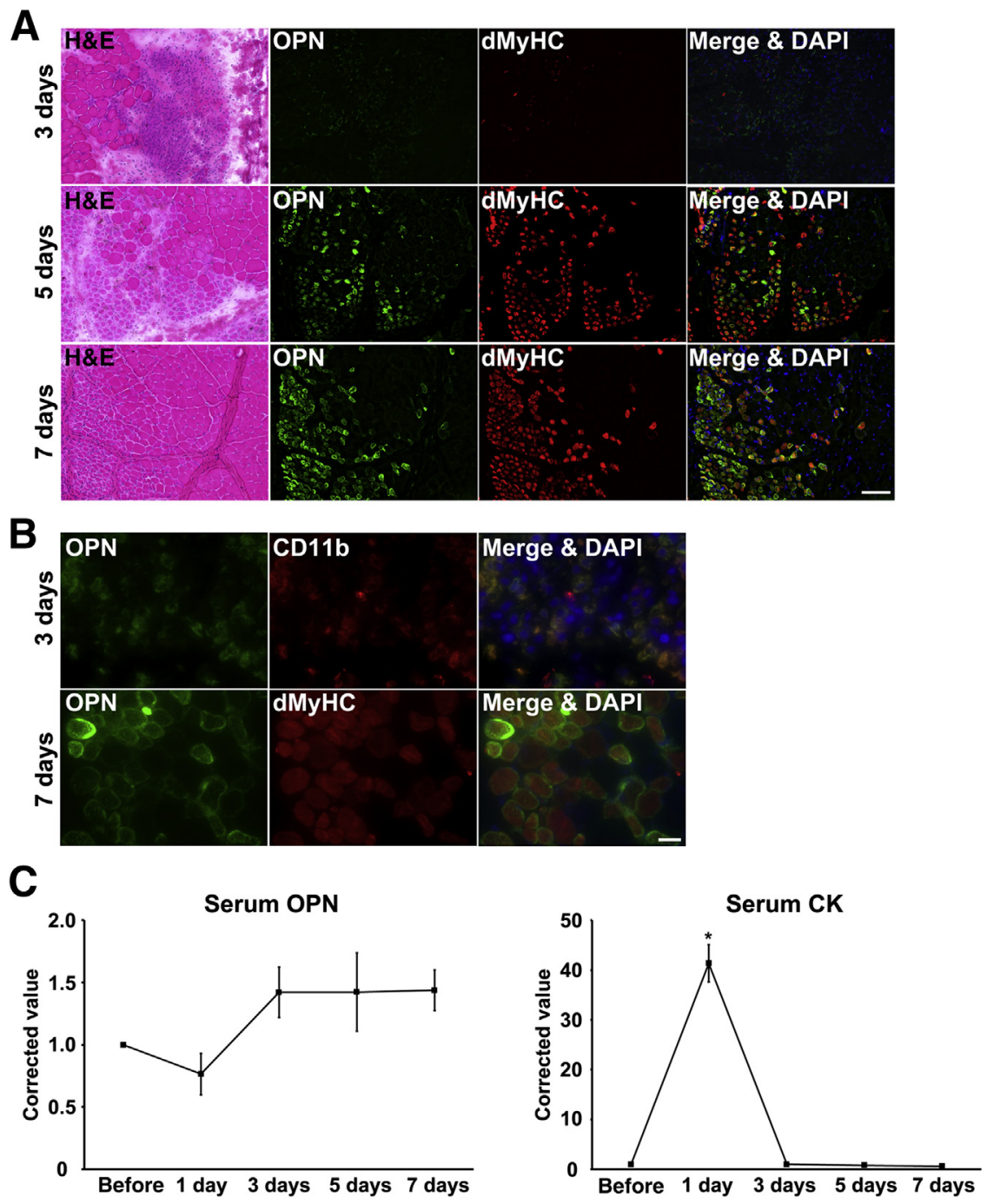

Figure 5 Osteopontin (OPN) expression during muscle regeneration after cardiotoxin-induced injury. A: Serial sections of tibialis cranialis (TC) muscles injured by cardiotoxin are stained with hematoxylin and eosin (H\&E) and immunostained for OPN (green) and developmental myosin heavy chain (dMyHC; red). Muscles from dogs at 3, 5, and 7 days after cardiotoxin injection. Merged images co-stained with DAPI (blue; nucleus) are shown on the right. B: High-magnification images of OPN expression are shown after immunostaining for CD11b at 3 days after injection or for $\mathrm{dMyHC}$ at 7 days after injection. Merged DAPI (blue; nucleus)-stained images are shown on the right. C: Serum OPN and creatine kinase (CK) levels in wild-type dogs before and after cardiotoxin injection. Each corrected preinjection and postinjection value of the same dogs was calculated by dividing by the preinjection value. Data are expressed as means \pm SEM. $n=9$ for before and after 1 and 3 days; $n=6$ for 5 days; $n=3$ for 7 days. ${ }^{*} P<0.05$ versus preinjection value using a paired $t$-test with the Holm multiple test. Scale bars: $100 \mu \mathrm{m}(\mathbf{A}) ; 20 \mu \mathrm{m}$ (B).

\section{OPN Expression in the Diaphragm and Limb Muscle at Birth}

We next performed immunostaining to observe OPN expression in WT and dystrophic muscle tissues around birth (Figure 2) because the serum OPN levels in dystrophic dogs revealed a distinct elevation pattern compared with serum CK. Histologic analysis of WT muscles revealed no impairment in muscle fibers at birth and after initial respiration. In the dystrophic diaphragms, we found a sporadic appearance of opaque muscle fibers before respiration (Figure 2A), whereas massive degenerative fibers were noted after respiration (Figure 2B), as previously reported. ${ }^{20}$ OPN expression was observed in infiltrating mononuclear cells, which were mostly CD11b-positive immune cells, including macrophages (Figure 2, A and B). Diffuse positive OPN staining was also observed in some interstitial cells and in small muscle fibers in WT and dystrophic muscles.

Histologic examination of TC muscles of dystrophic dogs revealed some opaque fibers both before and after respiration (Figure 2, A and B). In these dystrophic TC muscles, OPN was also expressed in CD11b-positive immune cells and in small-sized muscle fibers.

\section{Diaphragmatic and Limb Muscle OPN Expression at Different Ages}

We then examined the pathologic process in dystrophic muscles at different ages (Figure 3). No pathologic findings were observed in any of the WT muscles, and OPN was only detected in some interstitial cells. In the dystrophic diaphragms, degenerative muscle fibers accompanying infiltration of mononuclear cells were often observed at 3 weeks, 3.5 months, and 1 year of age. OPN was expressed in infiltrating CD11b- and CD18-positive macrophages or granulocytes but not CD3-positive $\mathrm{T}$ lymphocytes (Supplemental Figure S1). OPN-positive cells were partly co-localized with CD68-positive M1 and CD206-positive M2 macrophages. OPN was intensely stained in a subset of muscle fibers (Figure 3), suggesting regenerating muscle fibers. These OPN-positive fibers were frequently co-stained with $\mathrm{dMyHC}$, an immature and 

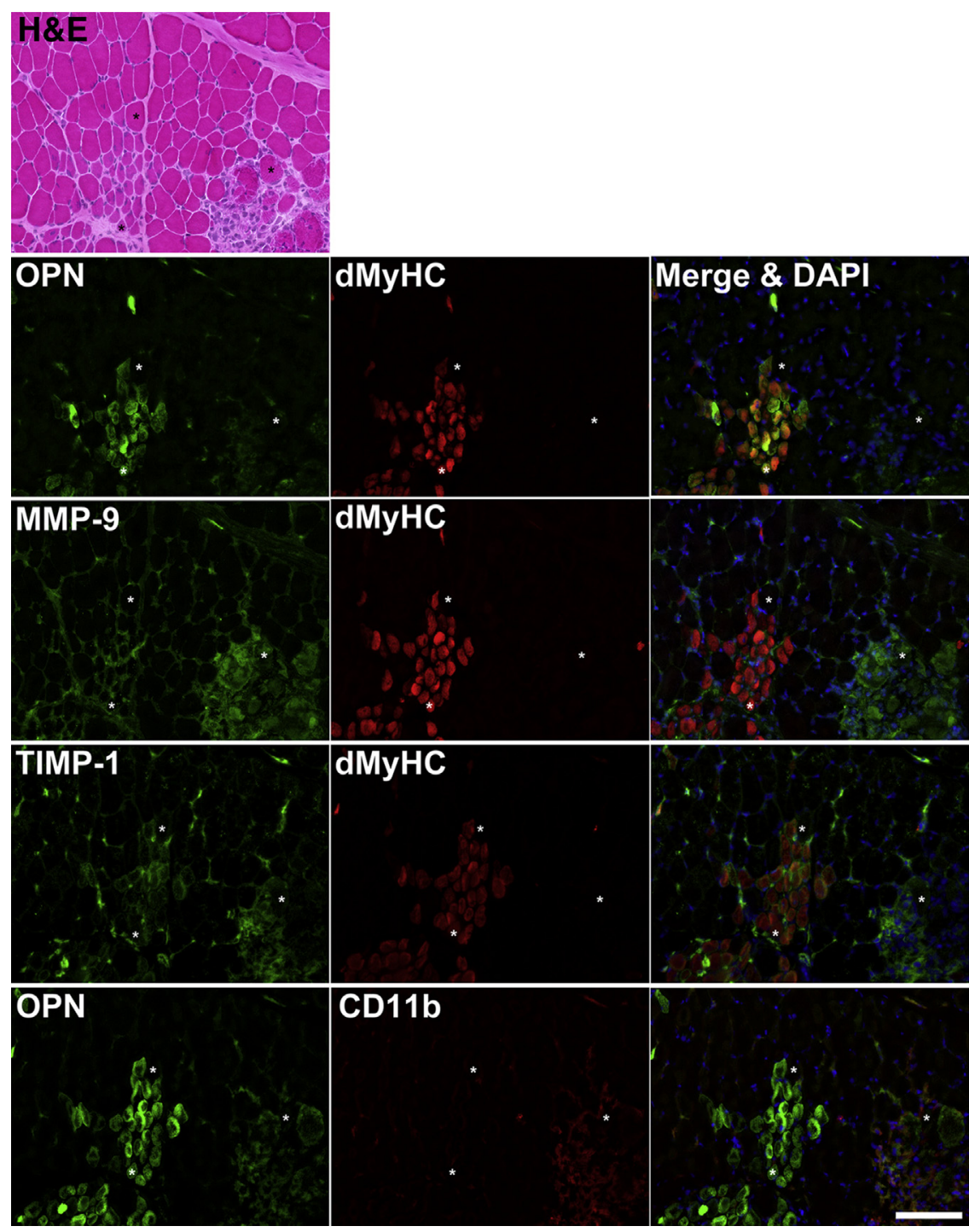

Figure 6 Expression of osteopontin (OPN), matrix metalloproteinase (MMP)-9, and tissue inhibitor of metalloproteinase (TIMP)-1 in dystrophic muscle. Serial sections of dystrophic tibialis cranialis (TC) muscle at the age of 3.5 months are stained with hematoxylin and eosin (H\&E) or immunostained for OPN, MMP-9, or TIMP-1 (green) and co-stained for developmental myosin heavy chain ( $\mathrm{dMyHC}$ ) or CD11b (red). Merged images that were co-stained with DAPI (blue; nucleus) are shown on the right. Asterisks indicate serial sections of the same muscle fibers. Scale bar $=100$ $\mu \mathrm{m}$. regenerating muscle fiber marker (Figure 4A). In these fibers, OPN was strongly expressed in the cytoplasm (Supplemental Figure S2). The histopathologic examination at the age of 2 years revealed fibrosis (Figure 3), and OPN was only faintly expressed in dMyHC-positive muscle fibers (Figure 3 and Figure 4A). In the dystrophic TC muscles, OPN was also expressed in infiltrating mononuclear cells and a subset of muscle fibers (Figure 3). OPN-positive fibers were co-stained with $\mathrm{dMyHC}$, as observed in the dystrophic diaphragm (Figure 4B).

To examine the proportion of OPN-positive immature and regenerating muscle fibers, we analyzed the percentage of OPN- and/or dMyHC-positive muscle fibers at different ages (Supplemental Figure S3). The percentages at each age range were not significantly different compared with that at the age of 2 to 4 years because of the small sample size. In the dystrophic diaphragm, the percentage of OPN and $\mathrm{dMyHC}$ double-positive fibers tended to be high at the age of 2 to 4 weeks, 3 to 5 months, and 1 year compared with that at the age of 2 to 4 years, whereas in the dystrophic TC muscle, the value tended to be highest at the age of 3 to 5 months.

\section{OPN Expression after Cardiotoxin Injection}

We analyzed OPN expression during muscle injury induced by cardiotoxin injection and subsequent regeneration in WT dogs (Figure 5). In the injured TC muscle tissues, mononuclear cells notably infiltrated in necrotic and edematous areas at 3 days after injection. Regenerating muscle fibers expressing dMyHC were increased at 5 and 7 days after injection (Figure 5A). Similar histologic features were observed in three WT dogs euthanized at each postinjection day. OPN was markedly expressed in CD11b-positive mononuclear cells and dMyHC-positive regenerating muscle fibers (Figure 5, A and B), indicating an association with muscle regeneration, as observed in dystrophic dogs. During muscle regeneration, serum OPN tended to increase, but no significant differences were seen at 3, 5, or 7 days after injection (Figure 5C). Serum CK elevation was observed at 1 day after injection, when substantial muscle injury was present. 


\section{Comparison of Tissue MMP-9 and TIMP-1 Expression} with OPN Expression

Different patterns of elevation were seen between serum MMP-9 and TIMP-1 levels in dystrophic dogs compared with serum OPN and CK. Thus, we performed immunostaining to detect these factors in dystrophic muscles (Figure 6 and Supplemental Figure S4). MMP-9 and TIMP-1 expression was observed in both the dystrophic diaphragm and TC muscles at the age of 3.5 months. MMP-9 was strongly expressed in infiltrating mononuclear cells, which were mainly CD11b- and CD18-positive macrophages or granulocytes (Supplemental Figure S4), and was not detected in dMyHCpositive muscle fibers (Figure 6). TIMP-1 was expressed in CD11b- and CD18-positive mononuclear cells and in dMyHC-positive regenerating muscle fibers. At the age of 2 years, the expression of MMP-9 and TIMP-1 was observed only in some interstitial cells (data not shown).

\section{Discussion}

We report that the serum OPN level in dystrophic dogs is elevated and reveals a different pattern compared with serum CK and MMP-9 levels and that this elevation pattern is related to muscle regeneration. Recently, OPN has been considered to be not only an inflammatory cytokine that functions in cellular adhesion and chemotaxis of immune cells, including macrophages, ${ }^{34,35}$ but also a myogenic factor that promotes myoblast migration, proliferation, and fusion into myotubes. ${ }^{11,12}$ Our results suggest that serum OPN can be a new biomarker of DMD that indicates muscle regeneration.

OPN was expressed in immune cells and a subset of regenerating muscle fibers of the dystrophic dog muscles, and a similar phenotype is also observed in patients with DMD. ${ }^{15}$ OPN is expressed in macrophages, fibroblasts, myoblasts, and myotubes during the muscle regeneration process after injury by intramuscular injection of snake venom. ${ }^{14}$ The up-regulation of OPN expression (6 to 48 hours and 3 to 14 days after venom) is correlated with two distinct phases that consist of the inflammatory response and muscle regeneration. These observations strongly suggest that $\mathrm{OPN}$ is an important mediator of muscle regeneration in the early phase. ${ }^{10}$ We also observed a higher percentage of OPN-positive regenerating muscle fibers for a longer period in the dystrophic diaphragm, where muscle regeneration is very active, ${ }^{36}$ compared with analysis of dystrophic TC muscles. Previous findings in our laboratory revealed that regenerating muscle fibers expressing slow-type myosin heavy chain are increased after the age of 4 months in the dystrophic dog diaphragm, whereas fast-type myosin heavy chain is predominant in the TC muscles. ${ }^{31}$ We speculate that OPN may be more closely related to slow-type muscle fibers than fast-type fibers during muscle fiber maturation, although further study is necessary.

In the present study, the serum OPN level in dystrophic dogs was elevated and revealed a different pattern compared with that of serum $\mathrm{CK}$, an established primary biomarker of DMD that indicates muscle injury. ${ }^{4}$ The serum CK level in dystrophic dogs was drastically elevated 1 hour after birth compared with the level just before birth, confirming our previous results that initial pulmonary respiration causes massive diaphragm injury in neonatal dystrophic dogs (Figure 2, A and B). ${ }^{20}$ Furthermore, we found that serum OPN was elevated in relation to muscle regeneration during the progressive phase, but not the chronic phase, when muscle regeneration is inactive. In mdx mice, the serum OPN level is increased at the ages of 4 and 16 weeks. ${ }^{13}$ Active immune cell infiltration and muscle regeneration are seen in muscle tissues in these $m d x$ mice, ${ }^{37,38}$ but whether the serum OPN level is related to muscle regeneration has not been verified. During the muscle regeneration process induced by cardiotoxin injection, OPN expression was present in infiltrating immune cells and regenerating muscle fibers, and serum OPN tended to increase. These results support our idea that serum OPN reflects the activity of muscle regeneration in skeletal muscles in dystrophic dogs.

Next, we compared the serum OPN levels to levels of serum MMP-9, an indicator of muscle inflammation, ${ }^{21-23}$ and its inhibitor TIMP-1 to further confirm whether serum OPN was indicative of muscle regeneration or inflammation. OPN contributes to increased amounts of MMP-9 as an immunomodulator, ${ }^{39}$ but the serum levels of these two factors were not similarly elevated in the dystrophic dogs. The serum MMP-9 level in dystrophic dogs was significantly increased 1 hour after birth compared with the level just before birth, and the pattern was similar to that of serum CK but not OPN. MMP-9 appears to rapidly reflect the inflammatory response, which can be explained by the following two reasons: the latent MMP-9 form (ie, deposited in the extracellular matrix) can be quickly converted to the active form through a proteolytic cascade, ${ }^{40}$ and MMP-9 can be immediately released from acutely infiltrating granulocytes due to storage of MMP-9 in the granules. ${ }^{41}$ In dystrophic dogs at the age of 3 months, both serum OPN and MMP-9 levels were significantly elevated compared with those in WT dogs. This consistent elevation actively reflects a serial change in muscle inflammation and regeneration. The elevation pattern of serum TIMP-1 was similar to that of serum OPN, and TIMP-1 expression was also observed in immune cells and regenerating muscle fibers in dystrophic muscles. Regarding the similar aspects of OPN and TIMP-1, these two factors may coordinately interact with each other in an MMP-independent manner. Indeed, TIMP-1 plays roles in the processes of cell growth, myogenesis, and fibrosis in addition to inhibiting MMP-9. ${ }^{25,26,42}$ However, serum TIMP-1 levels were not significantly different between dystrophic and WT dogs. Detection of serum OPN may be easier than detection of serum TIMP-1 in dystrophic dogs.

An important remaining question is whether we can use serum OPN as a novel biomarker in human DMD based on our dog results. The serum OPN level in dystrophic dogs was significantly correlated with the phenotypic severity at the onset of clinical signs, suggesting that serum OPN can 
be related to dystrophic disease in the early phase. In a previous study, the serum OPN levels were not significantly different between patients with DMD and controls, whereas the serum MMP-9 and TIMP-1 levels were significantly increased. ${ }^{21}$ The limitation of their study is that they included DMD patients with high mean ages $(13.1 \pm 6.2$ years; range, 2.9 to 31.2 years), and therefore, muscle regeneration was not expected to be active in those patients' muscles. Indeed, muscle regeneration in skeletal muscles of patients with DMD becomes inactive around the age of 9 years. ${ }^{43}$ We propose that the serum OPN level should be analyzed in young patients with DMD. We expect that serum OPN in patients with DMD can be used as a surrogate end point in clinical trials with a muscle regeneration inducer (eg, using granulocyte colony-stimulating factor), which improves the dystrophic phenotypes of utrophindystrophin double-knockout mice. ${ }^{44}$

Finally, the cleaved form of OPN, which is cleaved by thrombin or MMP-3, -7 , and -12 , actively regulates cell behavior and has high affinity for multiple integrin subtypes in other inflammatory and neoplastic disorders. ${ }^{6,8,9,45-48}$ The ratios of thrombin-cleaved versus noncleaved OPN in rheumatoid arthritis are significantly increased in plasma and synovial fluid compared with plasma from healthy controls. ${ }^{49}$ We attempted to distinguish cleaved and noncleaved forms of OPN in canine serum and plasma samples. However, we found that this distinction was difficult because we performed sandwich ELISA assay with two polyclonal anticanine OPN antibodies against unidentified epitopes, but anticanine OPN antibodies against the $\mathrm{N}$-terminal and $\mathrm{C}$-terminal halves were not available, and separation according to molecular weight in immunoblotting was not able to be optimized in the serum or plasma samples because of excessive amounts of albumin, which yielded close molecular weight to noncleaved form of glycosylated OPN, although we excluded serum or plasma albumin with commercially available kits. As a next step, identification of the cleaved and noncleaved forms of OPN in the plasma of patients with DMD could be feasible by ELISA with commercial antihuman OPN antibodies against the $\mathrm{N}$-terminal and C-terminal halves. Further analysis of each form could reveal the additional properties and roles of OPN in dystrophic disease.

In conclusion, serum OPN is a promising indicator of muscle regeneration in dystrophic dogs and may lead to the development of a novel biomarker for DMD. Moreover, serum OPN is expected to be applicable as a surrogate end point in clinical trials aimed at developing new treatments for DMD and other muscle disorders.

\section{Acknowledgments}

We thank Takashi Saito, Yumi Hayashita-Kinoh, Yuko Nitahara-Kasahara, Janek Hyzewicz, Ryoko Nakagawa, Naoki Ito, Naoko Yugeta, Masanori Kobayashi, Hironori Okada, Satoru Masuda, Chiaki Masuda, and Tomoko Chiyo for technical assistance and discussion and Hideki Kita, Shin-ichi Ichikawa, Yumiko Yahata-Kobayashi, Aya Kuriyama, Takayoshi Hikage, Akane Hanaoka, Namiko Ogawa, and other staff members of JAC Co. for their care of the dogs.

S.T. is the guarantor of this work and, as such, had full access to all the data in the study and takes responsibility for the integrity of the data and the accuracy of the data analysis.

\section{Supplemental Data}

Supplemental material for this article can be found at http://dx.doi.org/10.1016/j.ajpath.2016.01.002.

\section{References}

1. Sussman M: Duchenne muscular dystrophy. J Am Acad Orthop Surg 2002, 10:138-151

2. Emery AE: Population frequencies of inherited neuromuscular diseases-a world survey. Neuromuscul Disord 1991, 1:19-29

3. Matsumura K, Campbell KP: Dystrophin-glycoprotein complex: its role in the molecular pathogenesis of muscular dystrophies. Muscle Nerve 1994, 17:2-15

4. Emery AEH, Muntoni F: Duchenne Muscular Dystrophy, ed 3. Oxford, UK: Oxford University Press, 2003

5. Wang KX, Denhardt DT: Osteopontin: role in immune regulation and stress responses. Cytokine Growth Factor Rev 2008, 19:333-345

6. Morimoto J, Kon S, Matsui Y, Uede T: Osteopontin; as a target molecule for the treatment of inflammatory diseases. Curr Drug Targets 2010, 11: 494-505

7. Pagel CN, Wasgewatte Wijesinghe DK, Taghavi Esfandouni N, Mackie EJ: Osteopontin, inflammation and myogenesis: influencing regeneration, fibrosis and size of skeletal muscle. J Cell Commun Signal 2014, 8:95-103

8. Agnihotri R, Crawford HC, Haro H, Matrisian LM, Havrda MC, Liaw L: Osteopontin, a novel substrate for matrix metalloproteinase-3 (stromelysin-1) and matrix metalloproteinase-7 (matrilysin). J Biol Chem 2001, 276:28261-28267

9. Goncalves DaSilva A, Liaw L, Yong VW: Cleavage of osteopontin by matrix metalloproteinase-12 modulates experimental autoimmune encephalomyelitis disease in C57BL/6 mice. Am J Pathol 2010, 177: $1448-1458$

10. Hirata A, Masuda S, Tamura T, Kai K, Ojima K, Fukase A, Motoyoshi K, Kamakura K, Miyagoe-Suzuki Y, Takeda S: Expression profiling of cytokines and related genes in regenerating skeletal muscle after cardiotoxin injection: a role for osteopontin. Am J Pathol 2003, 163:203-215

11. Pereira RO, Carvalho SN, Stumbo AC, Rodrigues CA, Porto LC, Moura AS, Carvalho L: Osteopontin expression in coculture of differentiating rat fetal skeletal fibroblasts and myoblasts. In Vitro Cell Dev Biol Anim 2006, 42:4-7

12. Uaesoontrachoon K, Yoo HJ, Tudor EM, Pike RN, Mackie EJ, Pagel CN: Osteopontin and skeletal muscle myoblasts: association with muscle regeneration and regulation of myoblast function in vitro. Int J Biochem Cell Biol 2008, 40:2303-2314

13. Vetrone SA, Montecino-Rodriguez E, Kudryashova E, Kramerova I, Hoffman EP, Liu SD, Miceli MC, Spencer MJ: Osteopontin promotes fibrosis in dystrophic mouse muscle by modulating immune cell subsets and intramuscular TGF-beta. J Clin Invest 2009, 119:1583-1594

14. Barbosa-Souza V, Contin DK, Filho WB, de Araujo AL, Irazusta SP, da Cruz-Hofling MA: Osteopontin, a chemotactic protein with cytokine-like properties, is up-regulated in muscle injury caused by Bothrops lanceolatus (fer-de-lance) snake venom. Toxicon 2011, 58:398-409

15. Zanotti S, Gibertini S, Di Blasi C, Cappelletti C, Bernasconi P, Mantegazza R, Morandi L, Mora M: Osteopontin is highly expressed 
in severely dystrophic muscle and seems to play a role in muscle regeneration and fibrosis. Histopathology 2011, 59:1215-1228

16. Uaesoontrachoon $\mathrm{K}$, Wasgewatte Wijesinghe DK, Mackie EJ, Pagel CN: Osteopontin deficiency delays inflammatory infiltration and the onset of muscle regeneration in a mouse model of muscle injury. Dis Model Mech 2013, 6:197-205

17. Giacopelli F, Marciano R, Pistorio A, Catarsi P, Canini S, Karsenty G, Ravazzolo R: Polymorphisms in the osteopontin promoter affect its transcriptional activity. Physiol Genomics 2004, 20:87-96

18. Pegoraro E, Hoffman EP, Piva L, Gavassini BF, Cagnin S, Ermani M, Bello L, Soraru G, Pacchioni B, Bonifati MD, Lanfranchi G, Angelini C, Kesari A, Lee I, Gordish-Dressman H, Devaney JM, McDonald CM; Cooperative International Neuromuscular Research Group: SPP1 genotype is a determinant of disease severity in Duchenne muscular dystrophy. Neurology 2011, 76:219-226

19. Bello L, Piva L, Barp A, Taglia A, Picillo E, Vasco G, et al: Importance of SPP1 genotype as a covariate in clinical trials in Duchenne muscular dystrophy. Neurology 2012, 79:159-162

20. Nakamura A, Kobayashi M, Kuraoka M, Yuasa K, Yugeta N, Okada T, Takeda S: Initial pulmonary respiration causes massive diaphragm damage and hyper-CKemia in Duchenne muscular dystrophy dog. Sci Rep 2013, 3:2183

21. Nadarajah VD, van Putten M, Chaouch A, Garrood P, Straub V, Lochmuller H, Ginjaar HB, Aartsma-Rus AM, van Ommen GJ, den Dunnen JT, t Hoen PA: Serum matrix metalloproteinase-9 (MMP-9) as a biomarker for monitoring disease progression in Duchenne muscular dystrophy (DMD). Neuromuscul Disord 2011, 21:569-578

22. Kherif S, Lafuma C, Dehaupas M, Lachkar S, Fournier JG, VerdiereSahuque M, Fardeau M, Alameddine HS: Expression of matrix metalloproteinases 2 and 9 in regenerating skeletal muscle: a study in experimentally injured and mdx muscles. Dev Biol 1999, 205: $158-170$

23. Fukushima K, Nakamura A, Ueda H, Yuasa K, Yoshida K, Takeda S, Ikeda S: Activation and localization of matrix metalloproteinase-2 and -9 in the skeletal muscle of the muscular dystrophy dog (CXMDJ). BMC Musculoskelet Disord 2007, 8:54

24. Dahiya S, Bhatnagar S, Hindi SM, Jiang C, Paul PK, Kuang S, Kumar A: Elevated levels of active matrix metalloproteinase-9 cause hypertrophy in skeletal muscle of normal and dystrophin-deficient $\mathrm{mdx}$ mice. Hum Mol Genet 2011, 20:4345-4359

25. Sun G, Haginoya K, Chiba Y, Uematsu M, Hino-Fukuyo N, Tanaka S, Onuma A, Iinuma K, Tsuchiya S: Elevated plasma levels of tissue inhibitors of metalloproteinase- 1 and their overexpression in muscle in human and mouse muscular dystrophy. J Neurol Sci 2010, 297:19-28

26. Moore CS, Crocker SJ: An alternate perspective on the roles of TIMPs and MMPs in pathology. Am J Pathol 2012, 180:12-16

27. Shimatsu Y, Katagiri K, Furuta T, Nakura M, Tanioka Y, Yuasa K, Tomohiro M, Kornegay JN, Nonaka I, Takeda S: Canine X-linked muscular dystrophy in Japan (CXMDJ). Exp Anim 2003, 52:93-97

28. Shimatsu Y, Yoshimura M, Yuasa K, Urasawa N, Tomohiro M, Nakura M, Tanigawa M, Nakamura A, Takeda S: Major clinical and histopathological characteristics of canine X-linked muscular dystrophy in Japan, CXMDJ. Acta Myol 2005, 24:145-154

29. Yokota T, Lu QL, Partridge T, Kobayashi M, Nakamura A, Takeda S, Hoffman E: Efficacy of systemic morpholino exon-skipping in Duchenne dystrophy dogs. Ann Neurol 2009, 65:667-676

30. Hayashita-Kinoh H, Yugeta N, Okada H, Nitahara-Kasahara Y, Chiyo T, Okada T, Takeda S: Intra-amniotic rAAV-mediated microdystrophin gene transfer improves canine $\mathrm{X}$-linked muscular dystrophy and may induce immune tolerance. Mol Ther 2015, 23:627-637

31. Yuasa K, Nakamura A, Hijikata T, Takeda S: Dystrophin deficiency in canine X-linked muscular dystrophy in Japan (CXMDJ) alters myosin heavy chain expression profiles in the diaphragm more markedly than in the tibialis cranialis muscle. BMC Musculoskelet Disord 2008, 9:1

32. Holm S: A simple sequentially rejective multiple test procedure. Scand J Statist 1979, 6:65-70
33. Lin J, Jain S, Sun X, Liu V, Sato YZ, Jimenez-Fernandez S, Newfield RS, Pourfarzib R, Tremoulet AH, Gordon JB, Daniels LB, Burns JC: Lipoprotein particle concentrations in children and adults following Kawasaki disease. J Pediatr 2014, 165:727-731

34. Giachelli CM, Lombardi D, Johnson RJ, Murry CE, Almeida M: Evidence for a role of osteopontin in macrophage infiltration in response to pathological stimuli in vivo. Am J Pathol 1998, 152:353-358

35. Murry CE, Giachelli CM, Schwartz SM, Vracko R: Macrophages express osteopontin during repair of myocardial necrosis. Am J Pathol 1994, 145:1450-1462

36. Stedman H, Sweeney H, Shrager J, Maguire H, Panettieri R, Petrof B, Narusawa M, Leferovich J, Sladky J, Kelly A: The mdx mouse diaphragm reproduces the degenerative changes of Duchenne muscular dystrophy. Nature 1991, 352:536-539

37. Evans NP, Call JA, Bassaganya-Riera J, Robertson JL, Grange RW: Green tea extract decreases muscle pathology and NF-kappaB immunostaining in regenerating muscle fibers of mdx mice. Clin Nutr 2010, 29:391-398

38. Boettger T, Wust S, Nolte H, Braun T: The miR-206/133b cluster is dispensable for development, survival and regeneration of skeletal muscle. Skelet Muscle 2014, 4:23

39. Dahiya S, Givvimani S, Bhatnagar S, Qipshidze N, Tyagi SC, Kumar A: Osteopontin-stimulated expression of matrix metalloproteinase-9 causes cardiomyopathy in the mdx model of Duchenne muscular dystrophy. J Immunol 2011, 187:2723-2731

40. Michaluk P, Kaczmarek L: Matrix metalloproteinase-9 in glutamatedependent adult brain function and dysfunction. Cell Death Differ 2007, 14:1255-1258

41. Delclaux C, Delacourt C, D’Ortho MP, Boyer V, Lafuma C, Harf A: Role of gelatinase B and elastase in human polymorphonuclear neutrophil migration across basement membrane. Am J Respir Cell Mol Biol 1996, 14:288-295

42. Lewis MP, Tippett HL, Sinanan AC, Morgan MJ, Hunt NP: Gelatinase-B (matrix metalloproteinase-9; MMP-9) secretion is involved in the migratory phase of human and murine muscle cell cultures. J Muscle Res Cell Motil 2000, 21:223-233

43. Messina S, Vita GL, Aguennouz M, Sframeli M, Romeo S, Rodolico C, Vita G: Activation of NF-kappaB pathway in Duchenne muscular dystrophy: relation to age. Acta Myol 2011, 30:16-23

44. Hayashiji N, Yuasa S, Miyagoe-Suzuki Y, Hara M, Ito N, Hashimoto H, Kusumoto D, Seki T, Tohyama S, Kodaira M, Kunitomi A, Kashimura S, Takei M, Saito Y, Okata S, Egashira T, Endo J, Sasaoka T, Takeda S, Fukuda K: G-CSF supports long-term muscle regeneration in mouse models of muscular dystrophy. Nat Commun 2015, 6:6745

45. Scatena M, Liaw L, Giachelli CM: Osteopontin: a multifunctional molecule regulating chronic inflammation and vascular disease. Arterioscler Thromb Vasc Biol 2007, 27:2302-2309

46. Shojaei F, Scott N, Kang X, Lappin PB, Fitzgerald AA, Karlicek S, Simmons BH, Wu A, Lee JH, Bergqvist S, Kraynov E: Osteopontin induces growth of metastatic tumors in a preclinical model of nonsmall lung cancer. J Exp Clin Cancer Res 2012, 31:26

47. Yamaguchi Y, Shao Z, Sharif S, Du XY, Myles T, Merchant M, Harsh G, Glantz M, Recht L, Morser J, Leung LL: Thrombin-cleaved fragments of osteopontin are overexpressed in malignant glial tumors and provide a molecular niche with survival advantage. J Biol Chem 2013, 288:3097-3111

48. Kon S, Nakayama Y, Matsumoto N, Ito K, Kanayama M, Kimura C, Kouro H, Ashitomi D, Matsuda T, Uede T: A novel cryptic binding motif, LRSKSRSFQVSDEQY, in the C-terminal fragment of MMP3/7-cleaved osteopontin as a novel ligand for alpha9betal integrin is involved in the anti-type II collagen antibody-induced arthritis. PLoS One 2014, 9:e116210

49. Ohshima S, Yamaguchi N, Nishioka K, Mima T, Ishii T, UmeshitaSasai M, Kobayashi H, Shimizu M, Katada Y, Wakitani S, Murata N, Nomura S, Matsuno H, Katayama R, Kon S, Inobe M, Uede T, Kawase I, Saeki Y: Enhanced local production of osteopontin in rheumatoid joints. J Rheumatol 2002, 29:2061-2067 